\title{
Human T Cell Lymphotrophic Virus 1 After Transplantation: What Is the Risk of Recipient Disease?
}

\author{
D. R. Kaul* \\ Department of Internal Medicine, Division of Infectious \\ Disease, University of Michigan Medical School, Ann \\ Arbor, MI \\ *Corresponding author: Daniel R. Kaul,kauld@umich.edu
}

Received 07 February 2012, revised 10 February 2012 and accepted for publication 11 February 2012

Human T cell lymphotrophic Virus 1 (HTLV-1) is endemic in the Caribbean, parts of South America, West Africa, Asia and Oceania but rare in North America. After a prolonged latency, HTLV-1 is associated with development of acute T cell leukemia/lymphoma (ATL) in 2-5\% of infected individuals and HTLV-1-associated myelopathy (HAM) in a smaller percentage. Screening tests do not distinguish between HTLV-1 and HTLV-2 (not associated with disease) and most screen positive patients in nonendemic areas have HTLV-2 or a false positive result.

Before 2009, the Organ Procurement and Transplant Network (OPTN) required HTLV-1 testing on all deceased donors. Driven partly by the planned discontinuation in 2009 of the only FDA licensed screening assay practical for Organ Procurement Organization (OPO) use, an analysis of the risk/benefit of HTLV-1 screening was undertaken (1). This revealed that the prevalence of HTLV-1 infection was likely very low in potential organ donors (0.03-0.5\%). Despite the use of screen positive organs by selected centers with good short-term outcomes, most screen positive donors were not used and 167-227 organs were discarded annually. However, only $3-27 \%$ of these organs were likely infected with HTLV-1 (1). On the basis of this analysis, OPTN removed the requirement for universal deceased donor screening. Concern existed that the data used to make the above policy change was inadequate and that a period of retrospective testing would allow better determination of the true prevalence of HTLV-1 in organ donors, the risk of transmission and the expected natural history of donor-derived HTLV-1 infection. Largely because of practical concerns regarding retrospective testing, this proposal was not adopted.

The policy decision to discontinue universal donor HTLV-1 screening was not driven by the assumption that donor derived HTLV-1 infection will never occur; only that given poor performance of the test in a low seroprevalence populations, the risk benefit calculation did not favor continued testing. Thus, questions regarding HTLV-1 infection in solid organ transplantation (SOT) remain important (perhaps even more so in the absence of universal testing). Specifically, the three leading issues include understanding the risk of donor derived HTLV-1 disease, the natural history of HTLV-1 after SOT in a patient with pretransplant infection and the appropriate monitoring of infected/potentially infected recipients. In series that include long term follow up, few pretransplant HTLV-1 positive kidney transplant recipients develop ATL/HAM (2). Nonetheless, one series looking at cases of posttransplant lymphoproliferative disorder (PTLD) noted that 5 of 24 cases in kidney recipients were ATL in HTLV-1 positive patients suggesting that at least in some cases immunosuppression may hasten the development of HTLV-1 associated disease (3). Regarding donor derived HTLV-1 (a situation that might be higher risk than a positive recipient with some pretransplant immune response), one case report describes the relatively rapid development of HAM in three recipients of one HTLV-1 infected donor (4). Other reports describe possible donor derived HTLV-1 disease as well as asymptomatic probable donor derived infection.

The current report by Yoshizumi et al. (from an endemic area in Japan) provides some additional information regarding all of the above questions and raises additional concern regarding the risk of the development of serious HTLV1 disease in seropositive recipients (5). Combined with their previous report, this series represents the only report describing the natural history of HTLV-1 after liver transplantation. Over a 14 year period, 26/329 living donor liver transplants (LDLT) were performed on HTLV-1 positive recipients. Standard maintenance immunosuppression without cell-depleting induction and tapering to calcineurin inhibitor alone at 6 months was used. Fifteen percent (4/26) developed ATL (181-1315 days posttransplant); all died. One additional HTLV-1 negative recipient received a seropositive liver.

What does this report add to current knowledge of the approach to HTLV-1 seropositive potential recipients? Although a $\sim 15 \%$ chance of developing a usually fatal disease (with additional patients possibly developing neurological or malignant disease with further follow-up) is by 
no means low, surprisingly the overall survival did not differ between HTLV-1 positive and negative recipients. Thus, with careful informed consent, pretransplant recipient HTLV-1 infection should not be a contraindication to SOT. The use of HTLV-1 positive donors into seronegative recipients is more problematic. In low prevalence areas like North America, this is rarely an issue and perhaps only in extreme circumstances should HTLV-1 positive organs be transplanted into seronegative recipients. In circumstances where HTLV-1 donor status is positive (e.g. living donor), this report provides at least some additional comfort in that the single mismatched patient did not develop HTLV-1 disease after 6 years. This recipient remained seronegative but nucleic acid positive emphasizing that follow-up testing of seronegative recipients of seropositive donors should include both serological and nucleic acid testing. The elimination of the requirement for universal HTLV-1 deceased donor testing mandates that the transplant community remains vigilant for cases of HAM or ATL in recipients; this is likely the best way we have of determining the outcome resulting from discontinuation of donor testing.

\section{Disclosure}

The author of this manuscript has no conflict of interest to disclose as described by the American Journal of Transplantation.

\section{References}

1. Kaul DR, Taranto $\mathrm{S}$, Alexander $\mathrm{C}$, et al. Donor screening for human T-cell lymphotrophic virus 1/2: Changing paradigms for changing testing capacity. Am J Transplant 2010; 10: 207-213.

2. Tanabe K, Kitani R, Takahashi K, et al. Long-term results in human T-cell leukemia virus type 1-positive renal transplant recipients. Transplant Proc 1998; 30: 3168-3170.

3. Hoshida Y, Li T, Dong Z, et al. Lymphoproliferative disorders in renal transplant patients in Japan. Int J Cancer 2001; 91: 869-875.

4. Toro C, Rodés B, Poveda E, Soriano V. Rapid development of subacute myelopathy in three organ transplant recipients after transmission of human T-cell lymphotropic virus type I from a single donor. Transplantation 2003; 75: 102-104.

5. Yoshizumi T, Shirabe K, Ikegami T, et al. Impact of human T-cell leukemia virus type 1 in living donor liver transplantation. Am J Transplant 2012; 12: 1479-1485 